\title{
ANAEMIA
}

\section{Mutations in C5 explain eculizumab resistance}

Researchers recently reported

findings that explain the genetics underlying why certain Japanese patients with paroxysmal nocturnal haemoglobinuria (PNH) fail to respond to treatment with the anti-C5 antibody eculizumab, which has provided benefit to an overwhelming majority of patients (>95\%) of various ethnic origins.

Eculizumab inhibits the cleavage of complement protein C5 into C5a and C5b, preventing the formation of the membrane attack complex central to red blood cell lysis in PNH. In several clinical trials, the drug was shown to reduce intravascular haemolysis (measured using lactate dehydrogenase [LDH] reduction as a proxy), the need for transfusion and the risk of thromboembolic events, which are a major cause of mortality in patients with PNH. Despite these reported benefits, a small proportion of Japanese patients do not respond to eculizumab.

To explain this lack of response, researchers led by Jun-ichi Nishimura at the Osaka University Graduate School of Medicine studied blood samples from 11 poor responders. "We identified a C5 polymorphism in these patients with PNH," explains Nishimura. "This mutation prevents binding and blockade by eculizumab, while retaining the functional capacity of the mutant C5 to cause haemolysis." The missense mutation (c.2654G $\rightarrow$ A) was found in all 11 poor responders but not in seven responsive patients. A similar mutation (c.265C $\rightarrow$ T) was found in a poor-responding patient of Asian descent in Argentina. In screening people of Japenese, English, Scottish, Han Chinese and Mexican origin, 10 of 288 Japanese and only one of 120 Han Chinese individuals also harboured the mutation.

"Interestingly, some patients reported improvements in their quality of life or PNH-associated symptoms (including thrombotic tendency) despite their $\mathrm{LDH}$ being unaffected by eculizumab treatment," comments Nishimura.

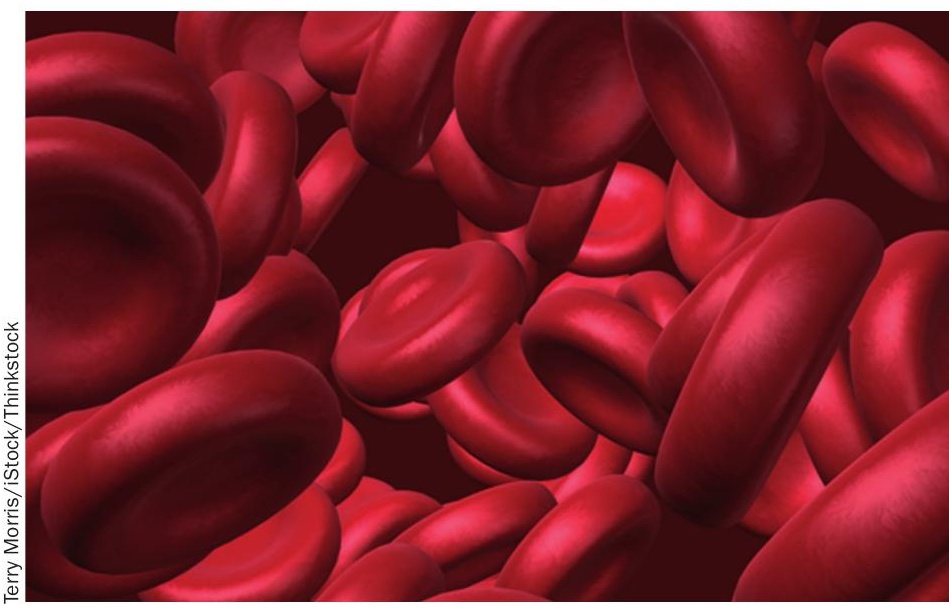

for C5 mutation, eculizumab could block the wild-type but not the structural variant $\mathrm{C} 5$, potentially reducing the amount of $\mathrm{C} 5 \mathrm{a}$ and $\mathrm{C} 5 \mathrm{~b}$ generated when the complement system is activated.

"This might be a possible reason for the improvement of PNH-associated symptoms."

The question that must now be addressed is whether patients with $\mathrm{PNH}$ who are of Japanese origin should be screened for mutations that will confer resistance prior to receiving therapy, and whether these patients can be offered alternative treatments. Nishimura and colleagues examined the effect of targeting C5 at a different site to that of eculizumab. They report that the haemolytic activity of serum samples from poor responders was suppressed by an anti-C5 antibody specific to a different epitope-namely, the N19-8 antibody. "Thus, the polymorphism in the target protein might be important to consider in patients with a poor response to the antibody-based treatments for various diseases," explain Nishimura.

\section{Mina Razzak}

Original article Nishimura, J. et al. Genetic variants in $\mathrm{C} 5$ and poor response to eculizumab. N. Eng. J. Med. doi:10.1056/ NEJMoa1311084 